\title{
COMMUNITY SECTOR ORGANISATIONS: A DIGITAL DIVIDE IN COMMUNICATION AND KNOWLEDGE CREATION?
}

\author{
Tom Denison \\ Centre for Community Networking \\ Monash University \\ Australia \\ Monash University \\ PO Box 197 \\ Caulfield East VIC 3145 \\ Email: Tom.Denison@sims.monash.edu.au
}

\begin{abstract}
In 2002, the Centre for Community Networking commenced a research programme aimed at improving the understanding of the use of communications and information technology (ICT) by community and third sector organisations within Australia. The immediate goal of this research was the creation of the Monash Community ICT Index. This paper represents the first attempt to provide a more in-depth analysis of the data obtained in that survey, setting the results in the context of research undertaken by Zook (2001), Castells (2001) and Adamic (2001), who consider that not only is the Internet a natural fit with "winner-take-all" markets but reinforces existing patterns of geographic centralisation in the information society and economy. It will pay particular attention to factors such as the location and size of organisations, and whether there is any support for the notion of a digital divide in the social communications networks and creators of knowledge within the Australian community sector.
\end{abstract}

\section{INTRODUCTION}

In 2002, the Centre for Community Networking (CCNR) at Monash University commenced a research programme aimed at improving our understanding of the use of communications and information technology (ICT) by community and third sector organisations within Australia. The immediate goal of this research was the creation of the Monash Community ICT Index, designed to be a national longitudinal data series providing indicators of use of ICT by community sector organisations, describing patterns of use and barriers. That survey was conducted by telephone between August 29 and the end of October 2002 and the initial findings have been made available (CCNR 2003).

The impetus for the research came from a perceived lack of general statistical information which adequately described the current Australian situation and a suitable theoretical framework in which to interpret it. The creation of the index was seen as having significant potential benefit for the public sector for planning, policy development and national co-ordination as well as providing a basis for further research and analysis. The Monash Community ICT Index itself was intended to provide a quantitative snapshot of the state of ICT usage within the Australian community sector. Future surveys should supplement it by providing evidence of trends, while further qualitative studies will allow not only exploration of questions of take-up and barriers to take-up but also the way in which community sector organisations operating in an online environment can make a positive contribution to social capital, defined by Putnam as the "features of social organisation, such as trust, norms and networks, that can improve the efficiency of society by facilitating coordinated actions" (Putnam et al. 1993, p. 167). As elsewhere, for example in the UK where the Office of the e-Envoy sees non-profits as important partners in the delivery of government services (2002), it is hoped that the research will inform government policy in the area.

In this paper, I explore one aspect of that broader research project, examining some of the research around the nature and development of the Internet, to determine whether there might be evidence of any environmental factors which may impinge on the development of Web sites by community sector organisations or their ability to use them effectively. I will argue that the interplay of external pressures and the nature of the Internet, particularly in regard to factors such as place and organisational size, can have a significant effect on the success of the Internet strategies of community sector organisations. I will also argue that the effective use of the Internet by most organisations is impeded by the presence of large sites which weaken the ability of smaller sites to promote themselves, so weakening their position with regard to their target audience. The consequences of these factors may well be the loss of local input and/or power and the placing of 
unrealistically high demands on sites because of the need to respond to external forces. Support for this will be garnered from a closer examination of the Monash Community ICT Index than has been made previously, but it must be seen as part of a broader research in progress. These questions will need to be explored in a qualitative sense in a series of future case studies, the design of which will be influenced by these findings.

\section{RESEARCH INTO THE NATURE OF THE INTERNET}

Despite the apparent potential of the Internet to enhance democratic participation and to eliminate place as a factor in information creation and distribution, there is a significant body of research that suggests that this is not occurring.

The recent work of Adamic and Huberman (2000, 2001), based on an analysis of America Online (AOL) log files, provides evidence that rather than leveling the playing field for content providers, the Internet strongly conforms to a power law distribution, typical of 'winner takes all' market models. While agreeing that the Internet's dispersal allows for greater accessibility to the content published by anyone, their results effectively demonstrate that patterns of usage follow a logarithmic scale, and at the two extremes, a handful of sites have millions of visitors while millions of sites attract only a handful of visitors. Notably, they found that $1 \%$ of all sites accounted for $55 \%$ of unique visits and, surprisingly, that when the analysis was restricted to .edu domains, the same distribution was found, with only slightly less drastic consequences $-5 \%$ of all sites accounted for $59 \%$ of unique users. The implication is that new sites will join the millions of 'invisible' sites and that, while some may advance over time, the majority will stay in the lower ranks. This sits well with the idea of early advantage and continuing dominance - high ranking sites are difficult but not impossible to dislodge, but even if the ranking of an individual site changes, the overall pattern remains the same. This suggests that the Internet tends toward reinforcing and increasing the gains of leading information creators and providers to the detriment of smaller players. It also has implications for all organisations trying to use the Internet, whether commercial or non-commercial in nature.

Adopting a different approach, Zook (2001) undertook a massive study based on the registration addresses for both CONE and CC domain names, on the assumption that this was the best method of determining the location of the production and organisation of Internet content. His results indicate that, far from being a dispersed medium, there is a striking centralisation of Internet publishers worldwide. In particular he notes that while the top 100 cities (only 46 of which are outside the US) contain $6.7 \%$ of the world's population they contain over half of the world's Internet domains. While he believes that it is too early to judge the long term pattern and economic impact, he argues that '1) the supply and demand for Internet content remains overwhelmingly within OECD countries; and 2) the production and supply of Internet content is primarily concentrated in the world's urban areas' (Zook 2001, p. 1680). Christie and Hepworth (2001), quoting research undertaken by Boldlygo.com in 2000, report a similar trend in the United Kingdom, where $80 \%$ of all dotcoms are based in south-east England (60\% in London alone) while other major centers such as Silicon Glen in Scotland and Silicon Fen in Cambridge accounted for a mere 4\% and 5\% respectively.

These findings support the notion not only that the Internet naturally favours the development of large-scale sites but that place, in the sense of the domination of key urban centres, is important. Characterising the Internet as a communications facilitator, Castells (2001) builds on this work by considering the digital divide from two perspectives - access to the Internet and control of information creation. He believes that in terms of access the digital divide is of diminishing significance but that these trends indicate that the question of control of information creation is much more problematic. He goes so far as to postulate that those who aren't a part of the Internet world will be marginalised and that, because of the nature of the Internet and its global and geographic characteristics, those who don't use it effectively will also be marginalised.

This is not as dramatic as it sounds - a focus on the structure can mask an ongoing rearrangement of the players and a cultural interplay that is not always one-way. For example, in discussing the music industry and its domination by five international conglomerates, Colista (1998) argues that while the 
overall structure of the sector has important consequences for the industry and the services it provides, it says little about the specific instances of cultural flow or changes to content that exist within that structure. 'Independent labels in the music industry may mediate some of the effects of such domination. These companies seek more innovative styles of music, often hoping to find the next big trend. Regardless of motivation, the existence of independent labels, which have also sought out international musics, serve as a source of diversity for listeners and as a recording vehicle for unknown acts' (Colista 1998, p.190). The importance of this is that in many ways the structure of the international music industry parallels that of the Internet and may provide useful insights into the consequences of that structure.

Nor is this a new phenomenon. Studies of the book industry document similar patterns of activity in both the $19^{\text {th }}$ and $20^{\text {th }}$ centuries, that is, centralisation and geographic concentration based on largescale mergers aimed at both economies of scale and market dominance, coupled with continual entry into the marketplace of numerous new small publishing houses which feed the cycle (Coser 1982, Lyons 2001). One important impact of this, which resonates strongly with the work of Adamic, is succinctly summarised by John Brooks, speaking for the Authors Guild, when he states:

It is not that fewer books are being published than formerly, or even, at least not provably, that books of exceptional merit are going unpublished. It is, rather, that such books are increasingly being squeezed out of sight by the conglomerate-hyped and -packaged best sellers, and not reaching the public that they deserve, and that deserve them. (Coser 1982, p. 29)

Again, there would appear to be a strong parallel with Internet publishing.

Why is this happening? Noam (2001) argues that the Internet thrives on economies of scale, and large organisations are best placed to achieve them. Elsewhere, he has made the claim that the 'Internet today is more concentrated than the rest of the information sector' (Noam 2003). Castells (2001) argues this is because early adopters set the cultural and social context for the Internet, and so dominate both the provision of information and the form of its communication. This assists in perpetuating their position. Adamic (2000) agrees that the early mover advantage is paramount and there is also support in standard texts on the information industry. For example, Varian and Shapiro (1999) argue that not only do early adopters indeed have an advantage overall but that these patterns are reflected, with distinct settings, within each major industry sector.

Another perspective is provided by research on the advantages of technological conglomerates such as technology parks which provide benefits through improved information flows, mutual learning and economies of scale. (Piore 1984). This also appears to be a factor in the success of broader geographic regions which are both strong in basic resources and can successfully harness aspects of social capital. For example, Silicon Valley's success vis a vis it's major competitor, the Route 128 corridor outside of Boston, is often claimed to be because of the informal horizontal networks established between industry players, whereas the Route 128 corridor relied on an 'I'll succeed on my own' philosophy, which resulted in poor performance in relative terms and its eventual eclipse. (Putnam 2000, Saxenian 1994). Thus, access to skills and a culture of sharing and innovation, based on close geographic proximity, would appear to provide a significant advantage in harnessing the potential of the Internet. If this is indeed the case, it would give a clear advantage to urban organisations.

Even if the overall concentration of Internet publishing is clear, the question remains as to whether this work is more related to business imperatives than those that drive community sector organisations. If it does apply, how does it manifest itself and what are the implications? After all, many community sector organisations do not want to be major institutions and are more interested in operating at the local level - but the question comes back to whether there are factors subjecting community sector organisations to the same pressures to enlarge, whether this is at the cost of local control and, from the point of view of the broader research, whether there are any consequences for social capital. 


\section{CHANGE, COMMUNITY SECTOR ORGANISATIONS AND THE INTERNET}

There is no doubt that community sector organisations are undergoing change. Within Australia, Lyons (2001) has demonstrated that there are many (often sector specific) reasons for this -such as the reduced membership base of trade unions and churches, changing demographics and the need for increased size in order to be able to work effectively with government. These pressures are not new. With regard to the use of IT specifically, an early ACOSS (1996) study into the use of IT by Australian community sector organisations made the point that many organisations are looking to IT so that they are able to enter into a partnership with government provision of community services on an equal footing. Increasingly this will mean taking up economies of scale in order to facilitate the adoption of e-commerce. Similar issues have been documented overseas, by Burt and Taylor (2002) in the UK, and Surman (2001) who notes the problems of fewer volunteers, decreased funding, increased need for coordination and higher demand for services in Canada. The community sector is undergoing changes and there are a number of pressures on organisations forcing them to attempt to expand, or at least reverse declines in their membership base. It is not unreasonable to assume that their use of the Internet would be seen as one opportunity to address some of the issues, and to assume that there will be some reflection of that in the type of presence they adopt, and the expectations of what that presence might achieve.

At a broader level, Giddens (1996) argues that the welfare state is under threat and at the very least undergoing restructuring and that 'the concept of a 'civil society' lying between the individual and the state... is a suspect one when applied to current social conditions' (Giddens 1996, p. 229). Both he and Castells (2001) consider that globalisation is posing both threats and opportunities and suggest that at the very least that community sector organisations are in the process of change and undergoing shifts in their structure. Certainly it is not hard to find other examples in this regard, which may require them to change in order to respond effectively e.g. the UN call for the World Summit on the Information Society on the one hand (WSIS 2003), and the growing criticism of nongovernment organisations by the new right on the other (Lobe 2003). These are global influences and certainly there will be pressure within countries to develop organisations that are capable of responding to these challenges. Once again, this implies larger organisations, groups of organisations or peak bodies that have sufficient resources to respond to the challenges.

Thus there are a number of external pressures on community sector organisations, at all levels, which are pushing towards a restructuring of the sector and which can be expected to have different consequences for organisations with different characteristics. Use of the Internet no doubt forms part of the response to these issues but, as can be seen, this will add to the pressure for larger organisations. The question requires further analysis and it may be that the impact on community sector organisations will be determined by a number of factors such as the size of organisation in question, its geographic location and constituency, and the nature of its activities. For example, the requirements of small localised groups with limited constituencies (e.g. the local kindergarten) should be simple enough for them to make effective use of the Internet without the need for economies of scale. Medium to large organisations with a more diverse geographic constituency may be forced into increasingly large operations in order to meet new challenges and benefit from the economies of scale necessary to achieve their objectives. Thus, they may be forced into competing with each other, adding to pressures for a restructuring of the sector.

This may well have a range of implications for the development of community sector organisations and their use of the Internet. It may also have implications for the maintenance of social capital. A pertinent example of how such restructuring may result in the atrophying of community life can be found in Heying's study (1997) of organisations in Atlanta. He argues that the economic transformations that occurred between 1961 and 1991, principally the delocalisation of corporate leadership consequent on the increasing nationalisation and globalisation of US business, broke the nexus of interlocking sectoral memberships by removing senior executives and the logic of their philanthropic contributions from 'place' to a more strategic way of thinking, leading to a decline in local civic contribution in Atlanta. In a similar vein, Putnam (2000) suggests that accelerating nationalisation and globalisation of US economic structures may be having a negative impact on levels of social capital - 'replacement of local banks, shops and other locally based firms by far- 
flung multinational empires often means a decline in civic commitment on the part of business leaders'. Being based on the US experience, these examples may reflect a stronger business/community nexus that has traditionally been the case in Australia, but they are nonetheless illustrative of the types of connections that exist and the ways in which they can be disrupted. This should be of significant concern to those interested in levels of social capital in rural and regional Australia, particularly when considering the effects of other developments, such as those illustrated by the economic modeling commissioned by the National Office for the Information Economy (NOIE). That work indicates that while the overall effect of e-commerce on regional Australia should be positive, it will come at the cost of significant restructuring, particularly in the area of local retail activity and banking presence (NOIE 2000). Add to this the potential for corresponding changes in the community sector, and the situation will require close monitoring.

In summary, it would appear that use of the Internet has real potential to reinforce, and perhaps exacerbate, existing patterns of activity, increasing the importance of place and the benefits of size, regardless of the type of organisation involved. Castells may be overstating the case that organisations will be marginalised because of the Internet, but it is true that community sector organisations face a range of pressures and that failure to make effective use of the Internet in response to those pressures only weakens their position. Harnessing the Internet in at least a partial response to these pressures may well reinforce their general thrust, and at a general level may well contribute to a delocalisation of the sector, with consequent losses in social capital. At a practical level - specifically in terms of the use of the Internet - this will have implications for the effectiveness with which Web sites can contribute to the goals of their organisations, and presumably the levels of satisfaction with their performance.

This analysis suggests a number of research questions which would need to be considered to determine whether there is any support on the ground for it. For example, is location an important factor? Are some community sector organisations better placed to take advantage of the Internet, and so respond to the challenges they face, based on where they are located? Is there evidence that particular community sector organisations are developing services so much faster and to such an extent that there control of the medium and domination of the field will lead to their expansion at the expense of smaller organisations and leading to a corresponding 'delocalisation' of those organisations, with a consequent decline in local social capital? These are very broad issues and would need qualitative studies and trend analysis to explore them thoroughly.

\section{MONASH COMMUNITY ICT INDEX}

That work remains to be done, but a closer look at the results of the Monash Community ICT Index should contribute to our understanding. As mentioned, this was a nationwide survey of community sector organisations, the definition of which was drawn from the work of the Australian Bureau of Statistics (ABS) and included 'any non-government, non-commercial organisation or group with a formal structure. It may be as large as a national charity or as small as a local book club. Purely ad hoc, informal and temporary gatherings of people do not constitute organisations...' (Australian Bureau of Statistics 2001). So, for example, community welfare organisations, sporting bodies, unions, business associations, churches, clubs, childcare centers, were included. Organisations and agencies directly funded by government, such as schools and public libraries, were excluded, as were large financial institutions such as credit unions.

The sample was drawn at random from two national directories - the Yellow Pages and that of OurCommunity.com. A total of 2,915 organisations were approached and 923 usable responses obtained, representing an overall response rate of $31.7 \%$ and an accuracy of $+/-4 \%$ at the $95 \%$ confidence interval. Drawing heavily on the model used in the ABS Business Use of IT surveys (2002), the survey aimed to collect base data about the take-up of communication and information technologies, and to provide information on the how well community sector organisations thought they were using those technologies to meet their needs. To that end, it was structured around the following broad questions:

- What proportion of community organisations currently use computers?

- Is that computer usage adequate to their needs? 
- What proportion currently have access to the Internet?

- Is that access adequate to their needs?

- What proportion currently publish a Web site?

- Does that Web site meet their needs?

- How will the situation vary over the coming 12 months?

The key findings of the survey together with the detail of the methodology have been published separately (CCNR 2003). Basically, the results confirmed trends identified in earlier surveys, (ACOSS 1996, VCOSS 1997), in terms of both the rapid take-up of communication and information technologies and the barriers to that take-up. For example, while the proportion of organisations using computers necessarily remained fairly static over the period at $97.1 \%$, the proportion with access to the Internet increased dramatically from approximately $40 \%$ to $89.7 \%$. The survey also found high levels of satisfaction with the performance of the technology. $77.8 \%$ of respondents agreed or strongly agreed with the statement that the "organisation's use of computers met their organisation's needs", rising to $84.9 \%$ when respondents commented on the situation expected in one year's time. Similarly, $76.5 \%$ of respondents agreed or strongly agreed that current Internet access was meeting the organisation's needs, rising to $85.6 \%$ when considering the situation a year into the future.

However, the findings relating to the publishing of Web sites were not as positive. The overall proportion publishing a Web site was found to be $61.1 \%$, with only $55.8 \%$ of those agreeing or strongly agreeing that the organisation's Web site was meeting its needs. Location was also found to be significant. Organisations located in the capital city of a state or territory were much more likely to have a Web site $-73.2 \%$ as compared to only $45.6 \%$ of those located in other areas. They were also more likely to be satisfied with the performance of their Web site $-59.7 \%$ of city-based organisations were satisfied as opposed to $48.6 \%$ of those in other areas. Significant differences also emerged in the capacity of organisations to adopt the technology when size of the organisation is taken into account. $49.1 \%$ of the smallest organisations publish Web sites as opposed to $77.6 \%$ of the largest. It is clear from Figure 1. that when a combination of these two factors, location and size, were taken into account, small (that is those with annual revenues of less than \$1million) regional organisations were found to be significantly less likely to publish a Web site.

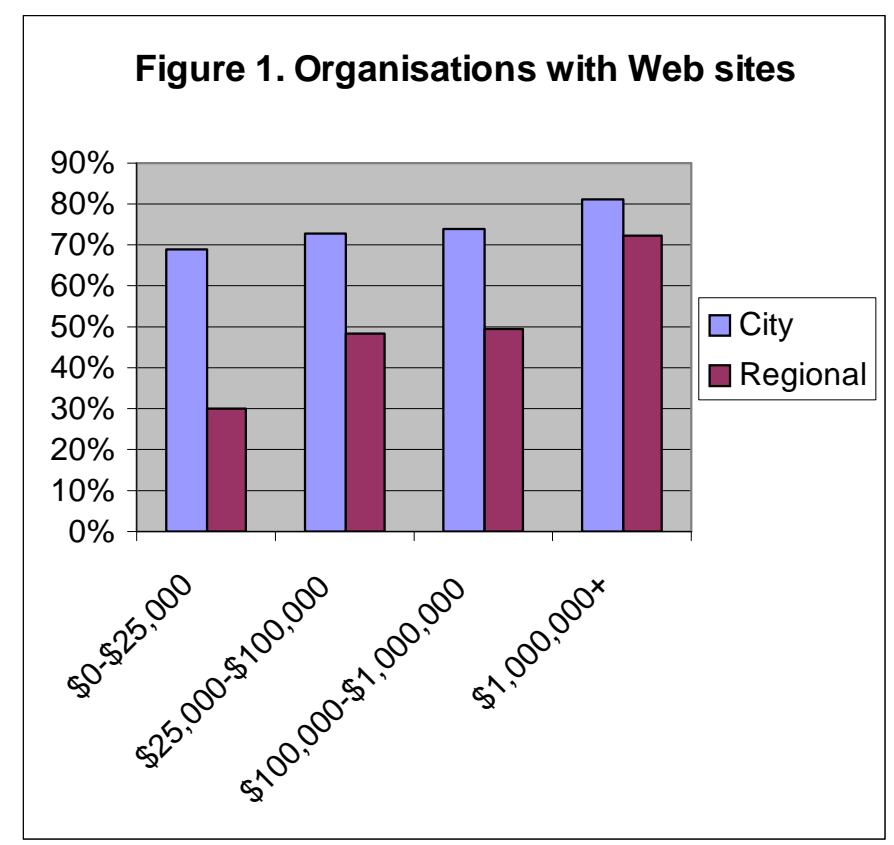


Overall, the reasons for not publishing a Web site were quite narrow. 28.7\% of organisations reported lack of skills as a significant barrier, followed by concerns about cost at $24.3 \%$. 26.8\% reported other concerns, and while these dealt with a variety of issues, most could be said to relate to resource issues and the current priorities of the organisations concerned. The number indicating that Web publishing was not suited to their organisation's needs was comparatively high at $35.7 \%$. Once again, the result obtained when the factors of size and location are combined is quite revealing: small regional organisations were least likely to have a Web site and lack of relevance to the organisation's needs was found to be the major factor, while for small city-based organisations it was the cost that was most important.

Some of the results relating to levels of satisfaction have been mentioned above, but in addition there was also found to be a significant relationship between the size of an organisation and levels of satisfaction - however, as can be seen from Figure 2., it was that the level of satisfaction with the Web site decreased with the increase in the size of the organisation: $67 \%$ of organisations with an annual revenue of less than $\$ 25,000$ pa were satisfied or very satisfied with their Web site, while the figure dropped to $42.7 \%$ for those organisations with revenue of $\$ 1$ million pa or more.

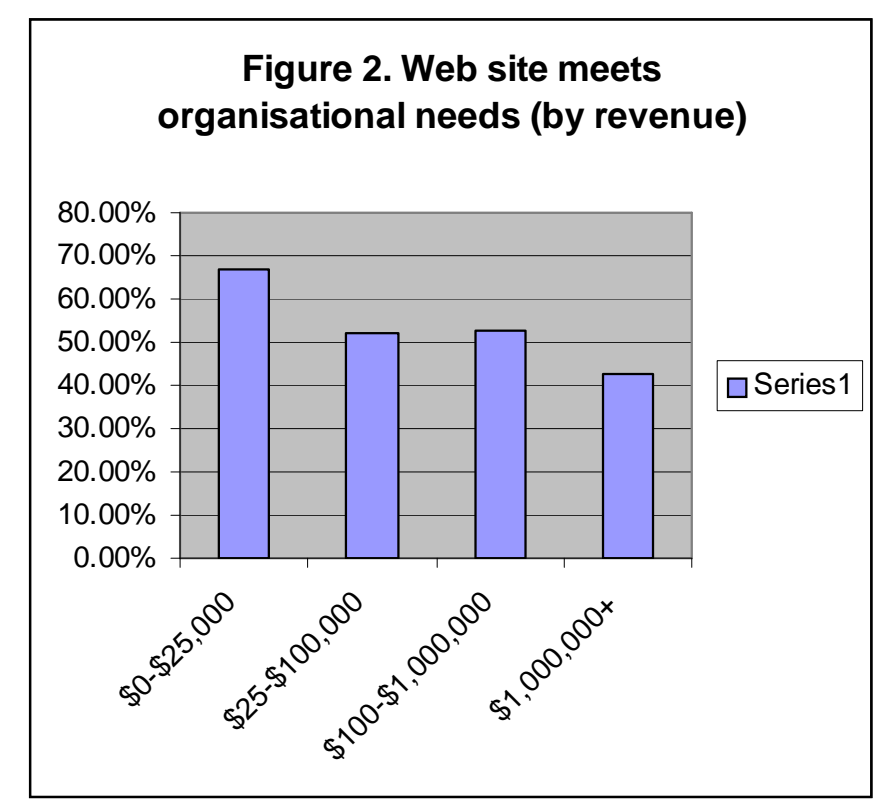

As can be seen from Figure 3., city-based organisations would appear to be better at taking advantage of the medium, and while the use of sites to collect information and to conduct financial transactions register as significant differences between city-based and regional organisations, the reality is that most organisations put their sites to a fairly limited range of uses. Related results include the fact that organisations with an annual revenue of over $\$ 1$ million are significantly more likely to use their Web site for financial transactions, and that organisations which provide access to online services or two-way communications (forums) on their sites are significantly more likely to be satisfied with the performance of their sites. 


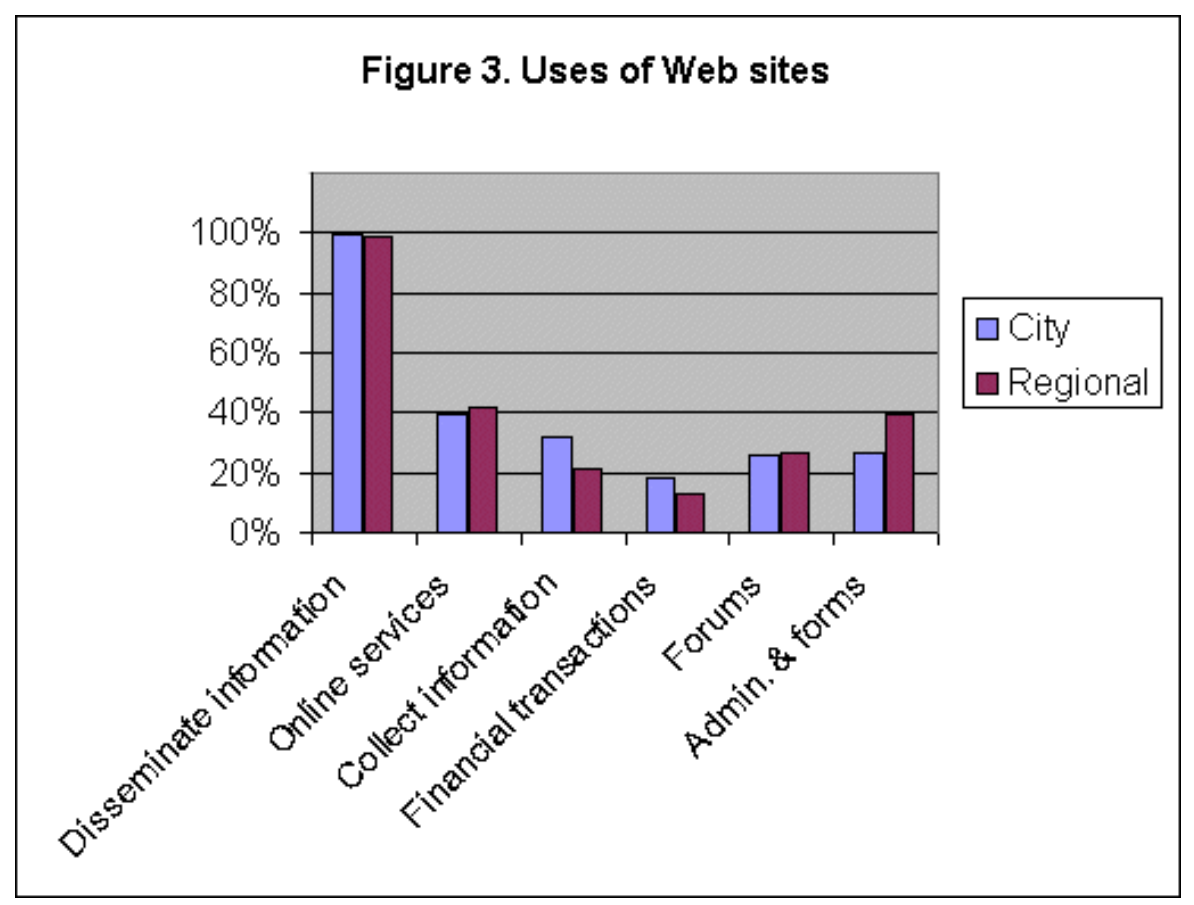

\section{CONCLUSION}

At first glance, many of these results would appear to support the analysis offered at the start of this paper. City-based organisations are significantly more likely to have Web sites and are significantly more likely to be satisfied with them. There is clearly a relationship between the size of an organisation and the likelihood that it will have a Web site - a pattern that holds true in both the cities and regions. Once again, city-based organisations are far more likely to have a Web site than regional organisations of comparable size, although the difference for the largest organisations is not significant. Finally, there is a definite inverse relationship between the size of an organisation and its level of satisfaction with its Web site, suggesting that perhaps the expectations on the performance of their Web sites is much higher. In the two major areas - location and size - the results are as suggested by the literature, and it would be easy, though premature, to accept that the mechanisms discussed earlier in this paper are at work. In fact, it may prove that these are only temporary divisions whose significance will diminish over time - that can only be established through the creation of a time series and further quantitative analysis. It may also be that there are other factors at work, and it will be the purpose of the previously mentioned case studies to explore that possibility, specifically by examining issues such as the aims of the organisation, any environmental issues it must deal with such as access to skills and innovation or pressures to enlarge, and the strategic aims of their Internet presence.

In conclusion, an examination of the research surrounding the nature of the Internet and the pressures on community sector organisations suggests that, in apparent opposition to its potential, the Internet is more likely to reinforce, and perhaps exacerbate, existing patterns of activity, increasing the importance of place and the benefits of size. While failure to make effective use of the Internet in response to those pressures will weaken their position, attempts to use it in at least a partial response to those pressures may well reinforce the general thrust of those pressures, and at a general level may well contribute to a delocalisation of the sector, with consequent losses in social capital. At a practical level - specifically in terms of the use of the Internet - this has implications for the effectiveness with which Web sites can contribute to the goals of their organisations, and presumably the levels of satisfaction with their performance. While an analysis of the results of the 
Monash Community ICT Index would appear to offer support for this argument, additional research must be undertaken in order to clarify the real situation.

\section{REFERENCES}

ACOSS (1996). Electronic Communication and the Community Sector, Final Report. ACOSS Paper No. 81, Australian Council of Social Services. (http://www.acoss.org.au/papers/current Accessed: 7/3/03)

Adamic, L. A., \& Huberman, B. A. (2000). The nature of markets in the World Wide Web. QJEC, 1(1): 5-12.

Adamic, L. A., \& Huberman, B. A. (2001). The Web's hidden order. Communications of the ACM, 44 (9): 55-58.

Australian Bureau of Statistics (2001). 4441.0 Voluntary Work, Australia.

Australian Bureau of Statistics (2002). 8129.0 Business Use of Information Technology

Burt, E. \& J. Taylor (1999). Information and Communication Technologies: Reshaping the Voluntary Sector in the Information Age? Centre for the Study of Telematics and Governance, Glasgow Caledonian University. (http://www.brunel.ac.uk/research/virtsoc/reports/voluntarymain.htm Accessed 23/3/03)

CCNR. (2003). The Monash Community Information and Technologies Index (CICT). School of Information Management and Systems, Monash Unversity: Clayton, Vic.

Castells, M. (2001). The Internet galaxy: reflections on the Internet, business and society. Oxford University Press: Oxford.

Christie, I. \& Hepworth, M. (2001). Towards the sustainable e-region. In Wilsdon, J. Digital futures: living in a networked world. (pp. 140-162) Earthscan Publications Ltd.: London.

Colista, C. \& Leshner, G. (1998). Traveling music: following the path of music through the global market. Critical Studies in Mass Communication, 15(2): 181-194.

Coser, L. A., Kadushin, C. \& Powell, W. W. (1982). Books: the culture \& commerce of publishing. Basic Books, Inc.: New York.

Giddens, A. (1996). In defence of sociology. Polity Press: Cambridge, UK.

Heying, C. H. (1997). Civic elites and corporate delocalisation: an alternative explanation for declining civic engagement. The American Behavioural Scientist, 40(5): 657-668.

Lobe, J. (2003). U.S. conservatives take aim at NGOs. CommonDreams. June 12, 2003. http://www.commondreams.org/headlines03/0612-09.htm (accessed 19/6/03).

Lyons, M. (2001). Third sector: the contribution of nonprofit and cooperative enterprises in Australia. Allen \& Unwin: Crows Nest, NSW.

Lyons, M. \& Arnold, J., (2001) A history of the book in Australia 1891 - 1945: a national culture in a colonized market. University of Queensland Press: St Lucia, Qld.

Noam, E. M. (2001). Will the Internet be bad for democracy? Talk - Camden, Maine, USA. http://www.citi.columbia.edu/elinoam/articles/int bad_dem.htm (accessed 10/6/2003).

Noam, E. M. (2003). The Internet: still wide open and competitive? Talk - Oxford Internet Institute 21/3/2003. http://www.oii.ox.ac.uk/seminar210303.html (accessed 10/6/2003).

NOIE (2000). E-commerce across Australia. National Office for the Information Economy: Canberra, ACT.

Office of the e- Envoy, U. (2002). E-enabling the Voluntary and Community sector. (http://www.eenvoy.gov.uk/publications/reports/e-enabling/summary.htm Accessed: 6/12/02)

Piore, M. J. \& Sable, C. F. (1984). The second industrial divide: possibilities for prosperity. Basic Books: New York.

Putnam, R., Leonardi, R. \& Nenetti, R. (1993). Making Democracy Work: Civic Traditions in Modern Italy, Princeton University Press: Princeton, NJ. Putnam, R. (2000). Bowling alone: the collapse and revival of American community. Simon \& Schuster: New York.

Saxenian, A. (1994). Regional advantage: culture and competition in Silicon Valley and Route 128. Harvard University Press: Cambridge, Massachussetts.

Surman, M. (2001). From Access to Applications: How the Voluntary Sector is Using the Internet.( http://www.volunteersonline.ca/news/environmentalscan.htm Accessed: $15 / 3 / 02)$. 
Varian, H. R. \& Shapiro. C. (1999). Information rules: a strategic guide to the network economy. Harvard Business School Press: Boston, Massachusetts, USA.

VCOSS (1997). Bridges and Barriers Research Project. The Council of Adult Education for the Victorian Council of Social Services.(http://www.skills.net.au/download/Bbrpt.doc Accessed: 7/3/03)

WSIS. (2003). World Summit on the Information Society. http://www.geneva2003.org/wsis/index_c01_2_07.htm (accessed 19/6/03).

Zook, M. A. (2001). Old hierarchies or new networks of centrality? - The global geography of the Internet content market. American Behavioural Scientist, 44 (10): 1679-1696. 